Full paper published in Noise and Fluctuations in Biological, Biophysical and Biomedical Systems, ed. S. M. Bezrukov, SPIE 66020X (2007).

\title{
The cardiorespiratory interaction: a nonlinear stochastic model and its synchronization properties
}

\author{
A. Bahraminasab, ${ }^{1}$ D. Kenwright,${ }^{1}$ A. Stefanovska, ${ }^{1}$ and P. V. E. McClintock ${ }^{1}$ \\ ${ }^{1}$ Department of Physics, Lancaster University, Lancaster, LA1 4YB, UK
}

We address the problem of interactions between the phase of cardiac and respiration oscillatory components. The coupling between these two quantities is experimentally investigated by the theory of stochastic Markovian processes. The so-called Markov analysis allows us to derive nonlinear stochastic equations for the reconstruction of the cardiorespiratory signals. The properties of these equations provide interesting new insights into the strength and direction of coupling which enable us to divide the couplings to two parts: deterministic and stochastic. It is shown that the synchronization behaviors of the reconstructed signals are statistically identical with original one. 